\title{
Lente intraocular multifocal refrativa: a performance visual e qualidade de vida em implantes bilaterais versus unilaterais
}

\author{
Refractive intra-ocular lens: visual performance \\ and quality of life between unilateral versus bilateral
}

Jorge Estefano Germano', Wilson Takashi Hida², Alexandre Almeida Brasil ${ }^{3}$, Renato Antunes Schiave Germano ${ }^{4}$, Iris de Souza Yamane ${ }^{5}$, Alexandre Reis ${ }^{6}$, Reinaldo Fujita ${ }^{7}$, Arthur Schaefer ${ }^{8}$, Newton José KaraJunior ${ }^{9}$, Amaryllis Avakian ${ }^{10}$, Celso Takashi Nakano ${ }^{11}$

\section{RESUMO}

Objetivo: O objetivo desse estudo é comparar acuidade visual, sensibilidade ao contraste, velocidade de leitura e a qualidade de vida pós-operatória de pacientes pseudofácicos unilaterais ou bilaterais com lentes intraoculares multifocais ReZoom. Métodos: Estudo comparativo, prospectivo, não aleatório incluindo 38 olhos de 24 pacientes, separados em um grupo com implante bilateral (14 pacientes) e outro com implante unilateral (10 pacientes). $\mathrm{O}$ exame oftalmológico completo incluiu acuidade visual não corrigida pré-operatória para perto, intermediário e para longe, acuidade visual com melhor correção, topografia corneal, sensibilidade ao contraste, velocidade de leitura e escala de satisfação realizado 60 dias após a cirurgia. A análise estatística foi realizada por meio dos testes de Kruskal-Wallis, Mann-Whitney e Qui-quadrado.

Resultados: Os resultados comparando função visual, sensibilidade ao contraste, velocidade de leitura e escala de satisfação foram semelhante entre os grupos. Conclusão: Não houve diferença estatística entre os resultados comparando função visual, sensibilidade ao contraste, velocidade de leitura e escala de satisfação entre os grupos.

Descritores: Presbiopia/cirurgia; Lentes intraoculares; Implante de lente intraocular; Acuidade visual

\footnotetext{
'Mestre responsável pelo departamento de catarata do Centro de Excelência em Oftalmologia de Bauru - Bauru (SP) - Brasil; ${ }^{2}$ Estagiário do setor da Catarata da Faculdade de Medicina da Universidade de São Paulo HC-FMUSP e Assistente do Setor da Catarata da Santa Casa de Misericórdia de São Paulo - SCMSP - São Paulo (SP), Brasil;

${ }^{3}$ Estagiário do setor da Catarata da Faculdade de Medicina da Universidade de São Paulo HC-FMUSP e Assistente do Setor da Catarata da Santa Casa de Misericórdia de São Paulo - SCMSP - São Paulo (SP), Brasil;

${ }^{4}$ Acadêmico do $4^{\circ}$ ano da Faculdade de Medicina da Universidade de São Paulo - USP - São Paulo (SP), Brasil;

${ }^{5}$ Estagiário do Departamento de Oftalmologia da Faculdade de Medicina da Universidade de São Paulo - USP - São Paulo (SP), Brasil; ${ }^{6}$ Estagiário do Departamento de Oftalmologia da Faculdade de Medicina da Universidade de São Paulo - USP - São Paulo (SP), Brasil ${ }^{7}$ Estagiário do Departamento de Oftalmologia da Faculdade de Medicina da Universidade de São Paulo - USP - São Paulo (SP), Brasil; ${ }^{8}$ Estagiário do Departamento de Oftalmologia da Faculdade de Medicina da Universidade de São Paulo - USP - São Paulo (SP), Brasil; ${ }^{9}$ Chefe assistente do Setor de Catarata do Departamento de Oftalmologia da Faculdade de Medicina da Universidade de São Paulo USP - São Paulo (SP), Brasil;

${ }^{10}$ Chefe assistente do setor da Catarata da Faculdade de Medicina da Universidade de São Paulo - USP - São Paulo (SP), Brasil;

${ }^{11}$ Chefe assistente do setor da Catarata da Faculdade de Medicina da Universidade de São Paulo - USP - São Paulo (SP), Brasil; Departamento de Oftalmologia da Faculdade de Medicina da Universidade de São Paulo - USP - São Paulo (SP), Brasil.
}

Recebido para publicação em: 11/2/2009 - Aceito para publicação em 11/6/2010 


\section{INTRODUÇÃO}

A cirurgia de catarata agregou nos últimos anos objetivos que anteriormente eram impensados, tornando a simples recuperação da visão para longe com correção óptica no pós-operatório obsoleta e insuficiente.

Convivemos com a realidade de uma facectomia com objetivos mais amplos, suprimindo por exemplo, a necessidade de correção para longe e até mesmo para perto $^{(1)}$

Tendo em vista tais expectativas, o objetivo do presente estudo foi avaliar e comparar a performance visual e a qualidade de vida de pacientes submetidos a implantes unilaterais e bilaterais de lentes intraoculares multifocais refrativas ${ }^{(2,3)}$.

\section{Métodos}

Este estudo foi conduzido de acordo com as normas éticas para pesquisa clínica e cirúrgica e foi aprovado pela Comissão de Ética para Análise de Projetos de Pesquisa (CAPPesq) da Diretoria Clínica do Hospital das Clínicas e da Faculdade de Medicina da Universidade de São Paulo, aprovação número 656/06, e com auxílio da Fundação de Amparo à Pesquisa do Estado de São Paulo (FAPESP).

Foi um estudo prospectivo, comparativo e não aleatório, no qual foram avaliados 38 olhos de 24 pacientes selecionados entre março de 2006 e outubro de 2008.

Os critérios de inclusão foram: idade entre 45 e 65 anos; presença de catarata primária bilateral; astigmatismo corneano menor que 1,00 dioptria em ambos os olhos; diâmetro pupilar de no mínimo $3,5 \mathrm{~mm}$ em condições mesópicas, medido pelo pupilômetro de Colvard (Oasis Corporation, Glendora, CA, USA); ausência de quaisquer outras doenças oculares, cirurgia ocular prévia, uso de medicações hipotensoras tópicas ou outras doenças sistêmicas que pudessem afetar o desempenho da visão pós-operatória, com diminuição da sensibilidade ao contraste, como a retinopatia diabética. Os pacientes que preenchiam os critérios de inclusão foram informados sobre a necessidade de exames complementares para medida das aberrações ópticas e sensibilidade ao contraste e somente foram incluídos aqueles que concordaram com a realização destes. Os critérios de exclusão foram: complicações per ou pós-operatórias, dúvidas quanto ao implante da LIO dentro do saco capsular ou descentração da LIO maior que $0,5 \mathrm{~mm}$ avaliada pelo exame à lâmpada de fenda.
A avaliação oftalmológica foi realizada em todas as visitas antes e depois da cirurgia e incluiu: acuidade visual com e sem correção para perto, intermédiaria e para longe ${ }^{(4)}$, medida da pressão intraocular e fundoscopia. As visitas após a cirurgia ocorreram no primeiro, terceiro, sétimo e nonagésimo dias de pós-operatório. Os pacientes foram submetidos à facoemulsificação convencional com implante da lente intraocular multifocal refrativa ReZoom ${ }^{\circledR}$ (Santa Ana, CA AMO $)^{(5)} 5$ em 38 olhos de 24 pacientes, separados em um grupo com implante bilateral (14 pacientes) e outro com implante unilateral (10 pacientes).

A lente intraocular (LIO) multifocal implantada trata-se de uma LIO refrativa com as seguintes características: zona óptica de acrílico hidrofóbico de $6 \mathrm{~mm}$ de diâmetro com bordas retas, 3 peças desenhada para o saco capsular, hápticos de polimetilmetacrilato (PMMA) em "C", diâmetro total de $13 \mathrm{~mm}$, disponível com poderes dióptricos entre $+6,0$ e $+30,0^{(2,6,7)}$.

A área multifocal dessa lente está contida em toda a extensão da parte óptica sendo cinco zonas anulares refrativas, basicamente arquitetadas da seguinte forma: a primeira zona (mais central), a terceira e a quinta (mais periférica) se destinam predominantemente à visão para distância e a segunda e a quarta zonas, à visão de perto. Essa disposição em zonas tem como intenção promover bom alcance visual nas variadas distâncias em distintas condições de iluminação, procurando conferir pupilo-independência. A asfericidade entre as zonas ópticas promove uma transição mais lenta e fisiológica objetivando melhorar a performance de visão intermediária. As zonas para visão de perto possuem adição de $+3,5 \mathrm{D}$, o que equivale a cerca de +2.57D no plano dos óculos e confere ao paciente ponto próximo a $39 \mathrm{~cm}^{(4,5,7,8)}$.

Tais características associadas ao conceito de que as zonas refratam os raios luminosos sem dispersão de energia corroboram o objetivo de reduzir a disfotopsia em condições mesópicas, bem como os halos em ambientes pouco iluminados e do ofuscamento em situações fotópicas $^{(9)}$.

O cálculo biométrico do candidato ao implante intraocular deve almejar uma refração final emétrope ou ligeiramente hipermetrópe para promover boa visão tanto de perto quanto de longe ${ }^{(10)}$.

Todos os pacientes selecionados tiveram poder dióptrico da LIO calculado pela técnica ultrassônica de imersão ${ }^{(11)}$ realizada por um único examinador experiente (J.G.), usando o biômetro Ocuscan RXP (Alcon Laboratories, Fort Worth, TX, USA) ou por interferometria de coerência óptica parcial com o biômetro IOL-Master (Carl Zeiss Meditec, Jena, 
Germany). As fórmulas usadas para selecionar o poder da LIO foram Hoffer-Q (comprimento axial menor que $22 \mathrm{~mm}$ ) e SRK/T (comprimento axial maior que $22 \mathrm{~mm}$ ), com refração alvo pós-operatória entre 0,1 e $0,4 \mathrm{DE}^{(10)}$.

Todas as cirurgias foram realizadas por um único e experiente cirurgião (J.G.), com técnica cirúrgica padronizada, que consistiu em: anestesia tópica; incisão de $2,75 \mathrm{~mm}$ em córnea clara, autosselante, no meridiano mais curvo; capsulorrexe; hidrodissecção com lidocaína a 1\% sem preservativos; uso de viscoelásticos dispersivo e coesivo pela técnica de "soft-shell" (12); facoemulsificação convencional e implante da LIO dentro do saco capsular, com injetor Emerald (AMO, Santa Ana, CA, USA). Foi prescrita, no período pós-operatório, a instilação de quinolona de quarta geração (gatiloxacina $0,3 \%$ ) por 10 dias, 4 vezes ao dia, associada a corticóide tópico (dexametasona $0,1 \%$ ) a cada 2 horas ao dia inicialmente e regredido progressivamente ao longo de 30 dias.

Todos os exames de medida de acuidade visual foram realizados monocularmente, com pupilas não dilatadas, com e sem a melhor correção óptica. A acuidade visual para longe $(6 \mathrm{~m})$, intermediária $(70 \mathrm{~cm})$ e para perto $(33 \mathrm{~cm})$ foi medida por meio da tabela de ETDRS (Precision Vision, Aurora, Colorado, USA) com conversão em logmar para longe e Jaeger para perto (Holladay VA conversion chart) sob condições controladas de luminosidade fotópicas estabelecidas em $85 \mathrm{~cd} / \mathrm{m} 2$.

A sensibilidade ao contraste foi medida com o VCTS ${ }^{\circledR} 6000$ (Vistech Consultants Incorporation, Dayton, $\mathrm{OH}, \mathrm{USA})$ sob condições fotópicas $(85 \mathrm{~cd} / \mathrm{m} 2)$ e mesópicas $(5 \mathrm{~cd} / \mathrm{m} 2)^{(4)}$. A tabela de medida da sensibilidade ao contraste apresenta linhas em cinco frequências espaciais entre 1,5 e 18 ciclos/grau (cpg). A base logarítimica 10 dos valores obtidos foi aplicada para se obter os valores de sensibilidade ao contraste que foram usados na análise estatística ${ }^{(13)}$.

Qualidade de vida foi definida levando em consideração a opinião subjetiva quanto ao conceito de qualidade ${ }^{(14)}$. Foi utilizado o questionário "TyPE questionnaire" que foi desenvolvido para avaliar a qualidade de vida após implante de lente intraocular multifocal, com objetivo de graduar a satisfação visual de 0 a 10 ( 0 ruim; 10 muito bom $)^{(1,3)}$.

A velocidade de leitura foi avaliada por meio da tabela "Minnesota Low Vision Reading Test" MNREAD (teste de acuidade de leitura Minnesota). Todos os testes foram feitos em condição binocular na distância de $40 \mathrm{~cm}$, com iluminação padronizada de 80 $\mathrm{cd} / \mathrm{m}^{2}$. A tabela MNREAD possibilita as seguintes medidas: a) acuidade de leitura: a menor letra que o paciente consegue ler sem cometer erros significantes; b) ta-

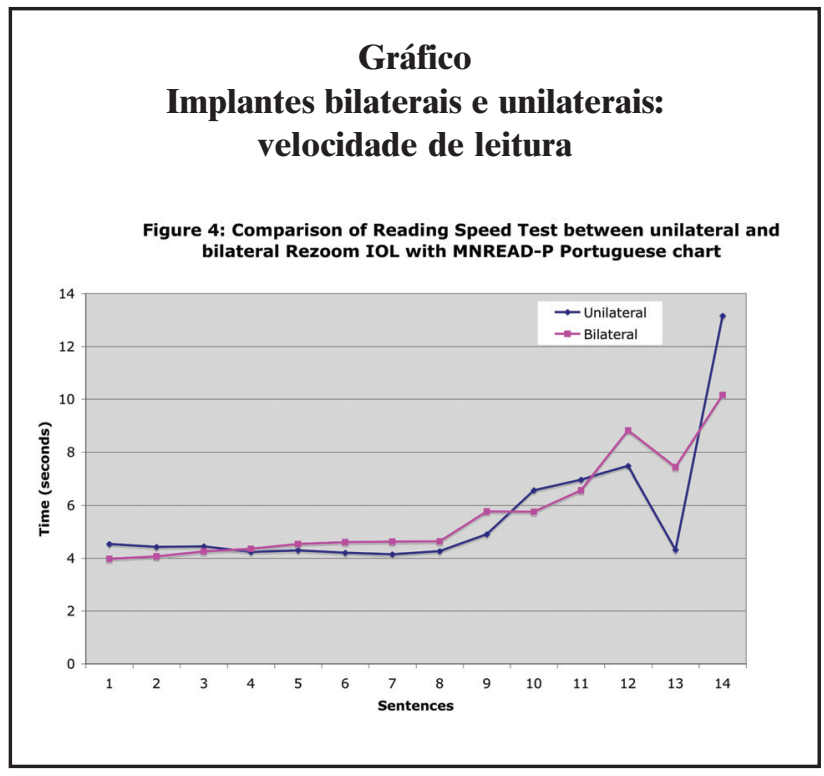

manho crítico de letra: tamanho mínimo de letra que o paciente consegue ler com máxima velocidade; e c) velocidade máxima de leitura: velocidade de leitura do paciente quando a leitura não está limitada pelo tamanho da letra ${ }^{(15)}$.

A significância foi testada ao nível de 5\% por meio dos testes de Kruskal-Wallis, Mann-Whitney e Quiquadrado (bicaudal), ajustando-se o nível de significância, quando necessário, para 1,7\%.

\section{Resultados}

O grupo com implante unilateral gerou como média em sua análise de satisfação 9.30 em relação à visão de longe, 7.90 à intermediária e 9.60 à de perto. Já o grupo com implante bilateral gerou como média 9.43 para a satisfação da visão de longe, 7.79 para a intermediária e 9.07 para a de perto (Tabela 1).

\section{Dıscussão}

As lente intraoculares multifocais têm representado um grande avanço na qualidade de vida e expectativa visual, principalmente no que se refere à independência do uso de óculos ${ }^{(2,6,7)}$. No presente estudo avaliamos a satisfação do paciente, além de melhor acuidade visual corrigida para longe, perto e intermediária.

Foi possível destacar a avaliação dos pacientes quanto a visão de longe e perto, com média superior a $9.0 \mathrm{em}$ ambos os grupos; corroborando com estudos anteriores, que também demonstraram a interessante 
performance descrita.

Há estudos sugerindo que, com pupila pequena e visao de longe a qualidade de imagem é significativamente melhor com a LIO refrativa comparada a outros tipos de LIOs difrativas. Já com pupilas maiores $(4.0 \mathrm{~mm}$ - 5.0mm) o efeito na visão de longe é semelhante entre LIOs multifocais difrativas e as LIOs refrativas. Entretanto isso ocorre as custas de perda de qualidade visual para perto ${ }^{(5,8)}$.

Os resultados de visão de longe são comparáveis a outros estudos. Os resultados de visão para perto são semelhantes quando o diâmetro pupilar atinge ao menos $3.5 \mathrm{~mm}^{(8)}$. Nessas condições é possível a utilização, pelo sistema óptico, da $2^{\circ}$ zona anular refrativa destinada para visão de perto.

Muitos artigos ressaltam a melhor performance da LIO em estudo, quando comparada a outras LIOs multifocais, no que concerne à visão intermediária; atribuída à transição asférica entre as zonas de foco de longe $(1,3$ e 5$)$ e as zonas de foco de perto $(2 \text { e } 4)^{(6,13)}$.

Apesar da LIO em estudo ter apresentado em outros estudos melhor visão intermediária comparado à LIO multifocal difrativa, os pacientes do presente estudo classificaram a visão intermediária como inferior comparado a visao de longe e perto conferida pela mesma $\mathrm{LIO}^{(4)}$.

Os pacientes submetidos ao implante bilateral atribuíram nota superior no questionário de satisfação visual comparado aos pacientes submetidos ao implante unilateral sobre a possibilidade de repetir o procedimento e ainda foram maiores entusiastas da possibilidade da realização da cirurgia com objetivo de substituir o uso de óculos. Foram também os pacientes com implante binocular, os que apresentaram menor queixa de ofuscamento. Esses dados sugerem a importância do implante bilateral da LIO multifocal em relação à qualidade da visão e à percepção da imagem pelo paciente, e à possibilidade de melhoria em sua qualidade de vida no que se refere à dependência dos óculos ${ }^{(16,17)}$.

Como era esperado, os pacientes submetidos ao implante bilateral da LIO apresentaram menor grau em dioptrias esféricas comparados aos pacientes submetidos ao implante unilateral. Foram também os pacientes deste grupo os que apresentaram melhor nota na avaliação sobre satisfação.

O grupo com implante unilateral gerou como média em sua análise de satisfação 9.30 em relação à visão de longe, 7.90 à intermediária e 9.60 à de perto. Já o grupo com implante bilateral gerou como média 9.43 para a satisfação da visão de longe, 7.79 para a intermediária e 9.07 para a de perto (Tabela 1).
Tabela 1

\begin{tabular}{|c|c|c|c|}
\hline & Bilateral & Unilateral & Valor de $p$ \\
\hline Perto $(33 \mathrm{~cm})$ & 9.07 & 9.60 & 0,95 \\
\hline Intermediária $(70 \mathrm{~cm})$ & 7.79 & 7.90 & 1,79 \\
\hline Longe (6 m) & 9.43 & 9.30 & 0,84 \\
\hline
\end{tabular}

Tabela 2

Implantes bilaterais e unilaterais: comparação das médias de dependência dos óculos

\begin{tabular}{lcc}
\hline Uso dos óculos & \multicolumn{2}{c}{ Rezoom (em \%) } \\
\cline { 2 - 3 } & Bilateral & Unilateral \\
\hline Computador & 42.86 & 28.57 \\
Jornal & 28.57 & 23.81 \\
Bula de remédio & 28.57 & 14.29 \\
Relógio de pulso & 47.62 & 47.62 \\
Livros & 33.33 & 19.05 \\
Cardápio & 28.57 & 19.95 \\
Fotografia & 28.57 & 28.57 \\
Dirigir à noite & 47.62 & 33.33 \\
Assistir televisão & 47.62 & 47.62 \\
\hline
\end{tabular}

Tabela 3

Implantes bilaterais e unilaterais: comparação do equivalente esférico residual (dioptrias)

\begin{tabular}{lcc}
\hline & Unilateral & Bilateral \\
\hline Média e desvio p & $-0,11 \pm 0,44$ & $-0,07 \pm 0,43$ \\
Variação & $-1,25 \mathrm{a}+0,25$ & $-1 \mathrm{a}+1,25$ \\
\hline
\end{tabular}

Tabela 4

Implantes bilaterais e unilaterais: comparação do equivalente cilíndrico residual (dioptrias)

\begin{tabular}{lcc}
\hline Equivalente cilíndrico & Unilateral & Bilateral \\
\hline Média e desvio padrão & $-0,41 \pm 0,54$ & $-0,17 \pm 0,52$ \\
Variação & $-1,5 \mathrm{a}+0$ & $-1 \mathrm{a}+1,5$ \\
\hline
\end{tabular}


Tabela 5

Implantes bilaterais e unilaterais: comparação das médias de acuidade visual com e sem correção para longe (LogMar)

\begin{tabular}{lccc}
\hline & & Bilateral & Unilateral \\
\hline Sem correção óptica & Média e desvio padrão & $-0,03 \pm 0,14$ & $-0,41 \pm 0,54$ \\
& Variação & $-0,2 \mathrm{a}+0,4$ & $-1,5 \mathrm{a}+0$ \\
Com melhor correção & Média e desvio padrão & $-0.07 \pm 0,13$ & $-0.07 \pm 0,13$ \\
& Variação & $-0,2 \mathrm{a}+0,4$ & $-0,2 \mathrm{a}+0,4$ \\
\hline
\end{tabular}

Consoante à possibilidade de ser submetido à cirurgia novamente, a média das notas atribuídas pelos pacientes foi de 9.30 para o grupo com implante unilateral e 8.79 para o grupo com implante bilateral. Quanto ao questionamento sobre ser submetido à cirurgia apenas para se tornar independente dos óculos, mesmo sem a presença de catarata, a média foi de 4.30 para os pacientes do grupo com implante unilateral e 6.71 para o bilateral. Os dois grupos apresentaram resultados semelhantes em relação ao uso dos óculos para ver as horas no relógio de pulso e fotografias e assistir à televisão

A queixa de ofuscamento foi relatada por $40 \%$ dos pacientes do grupo submetido ao implante unilateral e por $35.70 \%$ do grupo bilateral. A presença de halos à noite foi referida por $60 \%$ dos pacientes do grupo unilateral e por $71.42 \%$ dos pacientes do grupo bilateral. O resultado refrativo em relação ao equivalente esférico foi de $-0,11 \pm 0,44(-1.25 \mathrm{a}+0.25)$ naqueles em que o implante foi realizado em apenas um olho e de $0.07 \pm 0.34(-1.00 \mathrm{a}+1.25)$ naqueles em que o implante foi realizado em ambos os olhos.17

\section{Conclusão}

No presente estudo não houve diferença estatística entre os grupos com implante bilateral e unilateral da LIO Rezoom. Na literatura não constam trabalhos semelhantes, exceto pelo estudo que mostra equivalência na velocidade de leitura entre pacientes com LIO multifocal e olhos fácicos ${ }^{(18)}$.

Agradecimentos: Fundação de Amparo à Pesquisa do Estado de São Paulo (FAPESP - 656/06)

\section{Abstract}

Purpose: The aim of this study is to compare visual acuity, contrast sensitivity, reading speed and quality of life of postoperative pseudophakic patients with unilateral or bilateral ReZoom multifocal intraocular lenses. Methods: Comparative, prospective, not randomized study including 38 eyes of 24 patients were divided into a group with bilateral implantation (14 patients) and another with unilateral implantation (10 patients). The ophthalmological examination included visual acuity preoperative close, intermediate and far away, best corrected visual acuity, corneal topography, contrast sensitivity, reading speed and level of satisfaction achieved 60 days after surgery. Statistical analysis was performed using the Kruskal-Wallis, Mann-Whitney and Chi-square test. Results: The results comparing visual function, contrast sensitivity, reading speed and scale of satisfaction were similar between groups. Conclusion: There was no statistical difference between the results comparing visual function, contrast sensitivity, reading speed and level of satisfaction between the groups.

Keywords: Presbyopia/surgery; Lenses, intraocular; Lens implantation, intraocular; Visual acuity

\section{REFERÊNCIAS}

1. Javitt JC, Steinert RF. Cataract extraction with multifocal intraocular lens implantation: a multinational clinical trial evaluating clinical, functional, and quality-of-life outcomes. Ophthalmology. 2000;107(11):2040-8.

2. Lane SS, Morris M, Nordan L, et al. Multifocal intraocular lenses. Ophthalmol Clin North Am 2006;19(1):89-105, vi

3. Leyland MD, Langan L, Goolfee F, et al. Prospective randomised double-masked trial of bilateral multifocal, bifocal or monofocal intraocular lenses. Eye (Lond) $.2002 ; 16(4): 481-90$

4. Hida WT, Motta AF, Kara-Jose Junior N, et al. [Comparison between OPD-Scan results and visual outcomes of Tecnis ZM900 and Restor SN60D3 diffractive multifocal intraocular lenses]. Arq Bras Oftalmol. 2008;71(6):788-92.

5. Forte R, Ursoleo P. The ReZoom multifocal intraocular lens: 2-year follow-up. Eur J Ophthalmol. 2009;19(3):380-3.

6. Altmann GE, Nichamin LD, Lane SS, Pepose JS. Optical performance of 3 intraocular lens designs in the presence of decentration. J Cataract Refract Surg. 2005;31(3):574-85. 
7. Maxwell WA, Lane SS, Zhou F. Performance of presbyopiacorrecting intraocular lenses in distance optical bench tests. J Cataract Refract Surg. 2009;35(1):166-71.

8. Hida WT, Kara-Junior NJ, Nakano CT. Comparison of wavefront analysis between monofocal and multifocal posterior chamber implants. Can J Ophthalmol. 2008;43(2):248-9.

9. Montes-Mico R, Espana E, Bueno I, et al. Visual performance with multifocal intraocular lenses: mesopic contrast sensitivity under distance and near conditions. Ophthalmology. 2004;111(1):85-96.

10. Lavanya R, Teo L, Friedman DS, et al. Comparison of anterior chamber depth measurements using the IOLMaster, scanning peripheral anterior chamber depth analyser, and anterior segment optical coherence tomography. $\mathrm{Br} \mathrm{J}$ Ophthalmol. 2007;91(8):1023-6.

11. Olsen T, Nielsen PJ. Immersion versus contact technique in the measurement of axial length by ultrasound. Acta Ophthalmol (Copenh). 1989;67(1):101-2.

12. Auffarth GU, Holzer MP, Visessook N, et al. Removal times for a dispersive and a cohesive ophthalmic viscosurgical device correlated with intraocular lens material. J Cataract Refract Surg 2004;30(11):2410-4.

13. Hayashi K, Yoshida M, Hayashi H. All-distance visual acuity and contrast visual acuity in eyes with a refractive multifocal intraocular lens with minimal added power. Ophthalmology 2009;116(3):401-8.

14. Scott IU, Schein OD, West S, Bandeen-Roche K, Enger C, Folstein MF. Functional status and quality of life measurement among ophthalmic patients. Arch Ophthalmol 1994;112(3):329-35.
15. Castro CT, Kallie CS, Salomao SR. [Development and validation of the MNREAD reading acuity chart in Portuguese]. Arq Bras Oftalmol. 2005;68(6):777-83.

16. Chang DF. Prospective functional and clinical comparison of bilateral ReZoom and ReSTOR intraocular lenses in patients 70 years or younger. J Cataract Refract Surg. 2008;34(6):934-41.

17. Lacmanovic-Loncar V, Pavicic-Astalos J, Petric-Vickovic I, Mandic Z. Multifocal intraocular "mix and match" lenses. Acta Clin Croat. 2008;47(4):217-20.

18. Souza CE, Gerente VM, Chalita MR, Chalita MR, Soriano ES, Freitas LL, Belfort R, Jr. Visual acuity, contrast sensitivity, reading speed, and wavefront analysis: pseudophakic eye with multifocal IOL (ReSTOR) versus fellow phakic eye in non-presbyopic patients. J Refract Surg. 2006;22(3):303-5.

\section{Endereço para correspondência \\ Wilson Takashi Hida \\ Rua Afonso de Freitas, 488 apto 61 Paraíso \\ CEP: 04006 - 052}

São Paulo (SP), Brasil.

E-mail : witaks@yahoo.com.br 